\title{
PHACOEMULSIFICATION VERSUS ENDOCAPSULAR CATARACT EXTRACTION IN A UNIQUE COHORT OF PATIENTS
}

\author{
T. POTAMITIS ${ }^{1}$, S. BEATTY ${ }^{1}$, A. M. PEREIRA ${ }^{1}$ and J. L. PEARCE PEAR $^{2}$ \\ Birmingham and Redditch
}

\begin{abstract}
SUMMARY
The visual recovery and refractive results of 33 consecutive patients (66 eyes) undergoing standard endocapsular cataract extraction in one eye and simultaneous phacoemulsification in the fellow eye are reported. One surgeon performed all the operations. Surgically induced astigmatism was evaluated for the two techniques in terms of: (1) the proportion of eyes with a refractive cylinder of 1.5 dioptres $(D)$ or less; (2) the interocular difference in post-operative astigmatism. Eyes undergoing phacoemulsification had a significantly lower mean induced cylinder (paired Student's $t$-test: $t=3.729 ; p<0.001$ ) and were more likely to exhibit a cylinder of $1.5 \mathrm{D}$ or less (chi-squared test with Yates' correction: $\chi^{2}=7.88 ; p<0.01$ ) than the nuclear expression group. For paired eyes less postoperative astigmatism (Wilcoxon's signed rank test: $T=$ $92 ; p<0.01$ ) was seen in the phacoemulsification eye. At the time of the last post-operative assessment a significantly higher proportion of phacoemulsified eyes achieved a corrected Snellen visual acuity of $6 / 6$ or better than their fellow eyes (McNemar's test: $p<0.01)$. The results of this unique cohort of patients confirm the beneficial effects of phacoemulsification on astigmatism and visual outcome in the early postoperative period.
\end{abstract}

Phacoemulsification (PE) is rapidly becoming the preferred technique of cataract extraction for many ophthalmic surgeons. The speed of visual rehabilitation and the reduced post-operative astigmatism associated with this technique are the main factors contributing to the transition from conventional extracapsular cataract extraction (ECCE).$^{1-4}$

Simultaneous bilateral cataract extraction (SBCE) is a controversial subject and its risks, benefits and

\footnotetext{
From: ${ }^{1}$ Birmingham and Midland Eye Centre, Birmingham; ${ }^{2}$ Alexandra Hospital, Redditch, UK.

Correspondence to: T. Potamitis, Birmingham and Midland Eye Centre, Dudley Road, Birmingham B18 7QH, UK.
}

indications have been discussed elsewhere. ${ }^{5,6}$ It is not routinely performed in the developed world and consequently it has not been possible to compare the visual and refractive outcomes of PE and ECCE in the same patient over a single period of convalescence. In our study all surgery was performed by one surgeon (J.L.P.) who is a strong advocate of SBCE. We reviewed the visual and refractive results of 33 patients undergoing bilateral cataract extraction at one sitting. In each case one eye was arbitrarily assigned to undergo PE and the other eye conventional ECCE. The results are compared for the two techniques.

\section{PATIENTS AND METHODS}

Thirty-three patients underwent PE of one eye and simultaneous ECCE in the fellow eye. The mean age of the patients was 70.4 years with a range of 59-79 years.

A strict protocol for patient selection is used for simultaneous bilateral intraocular surgery. This includes: informed and valid consent; significant bilateral lens opacities; fitness for general anaesthesia (in no case was simultaneous bilateral cataract extraction performed under a regional block); proceeding to operate on the second eye only if the first was free of serious complications such as posterior capsule rupture with vitreous loss or expulsive haemorrhage. Patients with a predisposition to ocular infection (blepharitis, atopic eczema, trichiasis) or intraocular pathology that might prolong the procedure (posterior synechiae, pseudoexfoliation) are excluded. Pre-operative biometry was performed routinely, and in every case emmetropia was the desired post-operative refraction.

Data were retrieved from hospital records and all patients were reviewed and underwent refraction and keratometry by one of the authors. Astigmatism was expressed in terms of plus cylinder. Visual recovery was assessed using the unaided visual acuity 


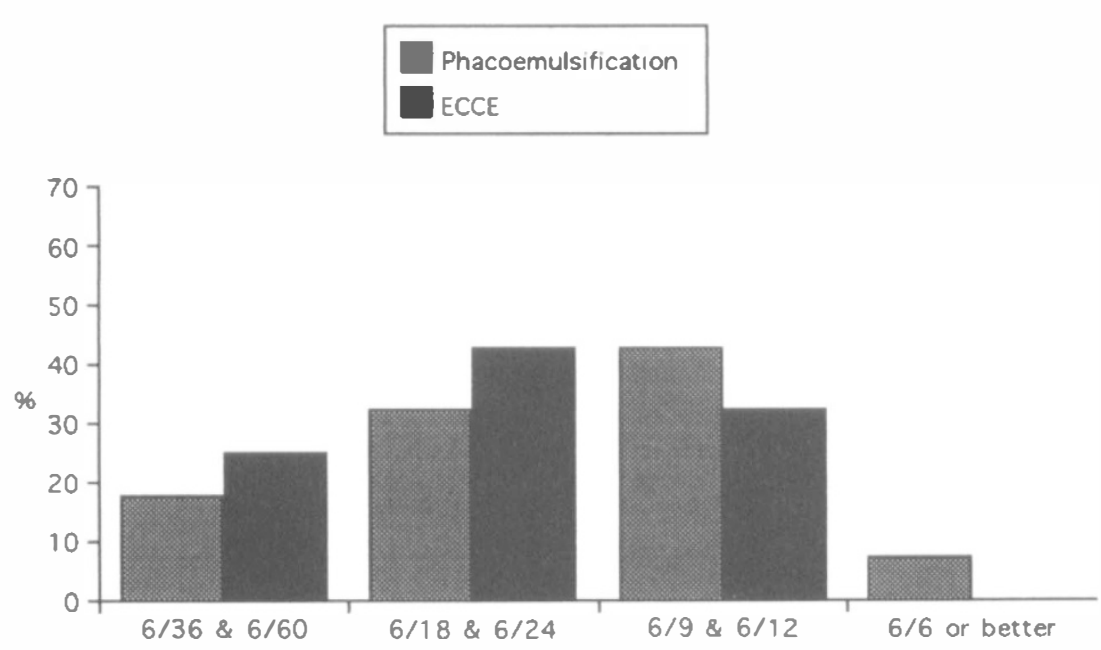

Fig. 1. Unaided visual acuity 1 week post-operatively.

at 1 and 8 weeks post-operatively and corrected visual acuity at the time of the last assessment. In addition, every patient was asked if they had a preference for either eye in terms of visual recovery. In our analysis of visual outcomes and ocular preference, patients with functional visual disability not fully accounted for by cataract formation were excluded.

We used the paired Student's $t$-test, the chisquared test (with Yates' correction where appropriate), Wilcoxon's signed rank test and McNemar's test in our statistical analysis.

\section{Surgical Technique}

Conventional Extracapsular Surgery. An $8-10 \mathrm{~mm}$ superior limbal incision was made and sodium hyaluronate injected into the anterior chamber. An envelope capsulotomy was then performed. The nucleus was expressed using the bimanual technique. Cortical clean-up was achieved using the co-axial irrigation-aspiration manual system. Sodium hyaluronate was then injected into the bag and a $7 \mathrm{~mm}$ optic one-piece polymethylmethacrylate (PMMA) intraocular lens (IOL) inserted. The anterior capsulotomy was then completed. The wound was sutured with a continuous 10-0 nylon suture and all the sodium hyaluronate aspirated from the anterior chamber.

Phacoemulsification Surgery. A $3.2 \mathrm{~mm}$ biplanar incision was made in temporal clear cornea and sodium hyaluronate injected into the anterior chamber. A manual capsulorhexis was performed and this was followed by hydrodissection of the lens. The nucleus was phacoemulsified in the posterior chamber using the 'divide and conquer' technique. Cortical clean-up was achieved by irrigation with balanced salt solution and automated aspiration. The capsular bag was filled with sodium hyaluronate and after extending the incision a $5.5 \mathrm{~mm}$ optic one-piece PMMA IOL was inserted. The wound was not sutured and the sodium hyaluronate was aspirated from the anterior chamber at the end of the procedure.

\section{RESULTS}

Post-operative follow-up ranged from 24 to 53 weeks

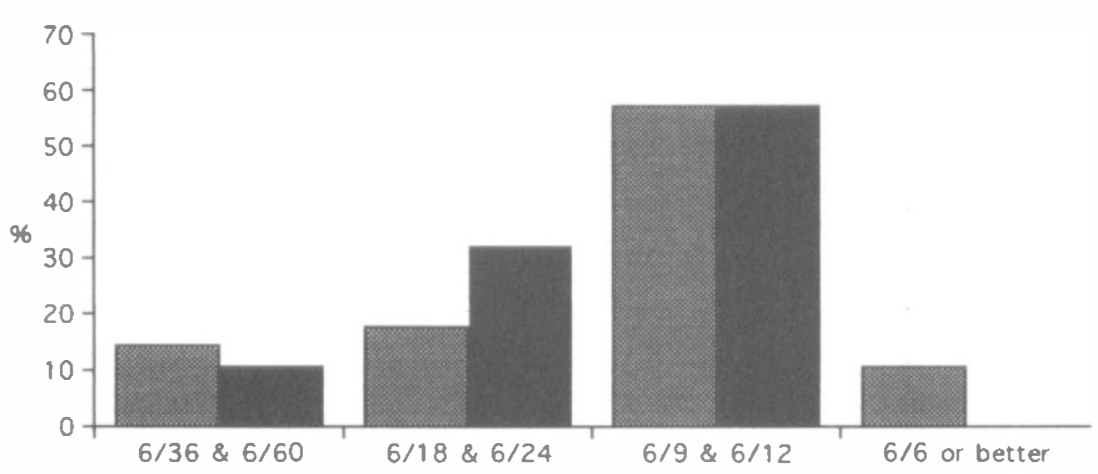

Fig. 2. Unaided visual acuity 8 weeks post-operatively. 


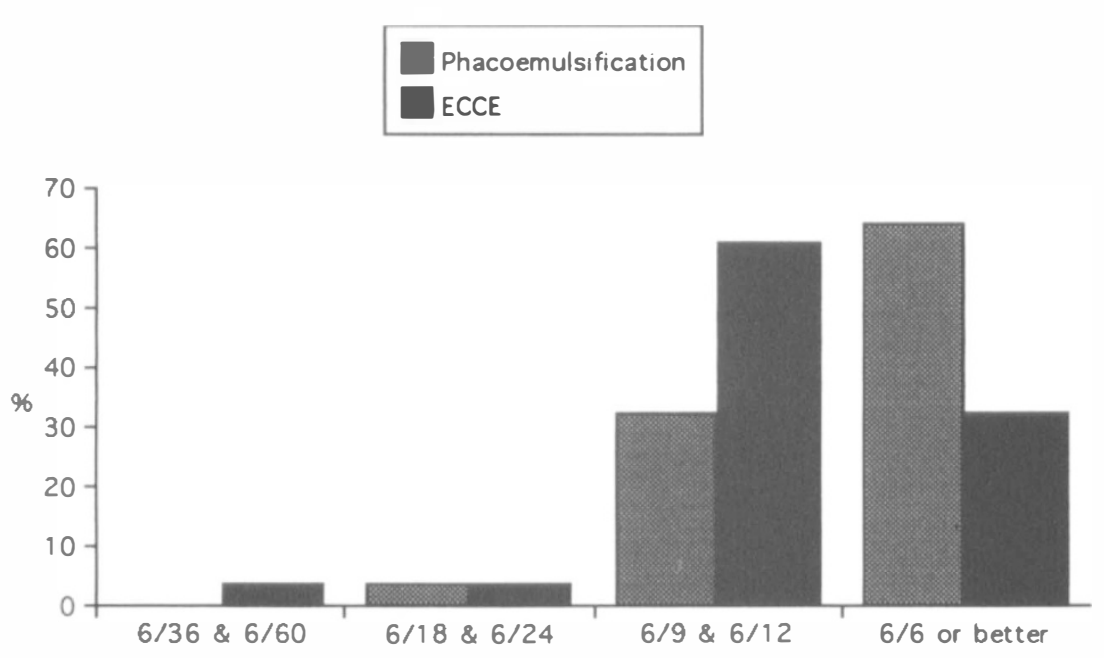

Fig. 3. Best corrected final visual acuity.

(mean 35 weeks). Two patients were lost to followup after the 4 week post-operative visit and were therefore excluded. Coexisting ocular pathology was recorded in 1 eye of 3 patients and included central retinal vein occlusion, branch retinal vein occlusion and age-related macular degeneration. These patients were therefore excluded from our analysis of visual recovery. No intra- or post-operative complications were recorded.

One week post-operatively an unaided visual acuity of $6 / 12$ or better was achieved in 14 of $28 \mathrm{PE}$ eyes $(50 \%)$ compared with 9 of the paired eyes which underwent ECCE (32.1\%) (Fig. 1). This difference did not reach statistical significance (McNemar's test: $p>0.3$ ). By the eighth post-operative week this level of uncorrected acuity was recorded in $19(67.8 \%)$ of the PE eyes and $16(57.1 \%)$ of the ECCE eyes (p>0.3) (Fig. 2).

A best corrected final visual acuity of $6 / 12$ or better was recorded in $27 \mathrm{PE}$ and 26 ECCE eyes (Fig. 3) $(p>0.3)$. However, a significantly higher proportion of eyes undergoing PE achieved a corrected Snellen acuity of $6 / 6$ or better (17 PE eyes, 9 ECCE eyes; $p<0.01$ ).

When asked to state a preference for one of the two operated eyes, 20 patients $(71.5 \%)$ chose the PE eye.

Thirty-one pairs of eyes were considered in our analysis of post-operative astigmatism. Of these, the PE eye had less astigmatism than the ECCE eye in 25 cases $(80.5 \%$; Wilcoxon's signed rank test: $T=92$; $p<0.01)$. An interocular difference in post-operative corneal cylinder of more than $1 \mathrm{D}$ was seen in 21 patients. In this group, the eye with the higher cylinder had undergone PE in 4 cases and ECCE in 17 cases $\left(\chi^{2}=16.29 ; p<0.001\right)$.

The mean post-operative cylinder for $\mathrm{PE}$ was significantly lower than for ECCE (PE, $1.24 \pm$ $0.833 \mathrm{D}$; ECCE, $2.21 \pm 1.27 \mathrm{D}$; paired Student's $t$ test: $t=3.729 ; p<0.001)$. A post-operative cylinder of greater than $1.5 \mathrm{D}$ was significantly more frequent in the ECCE group (10 eyes; 32.2\%) than among eyes
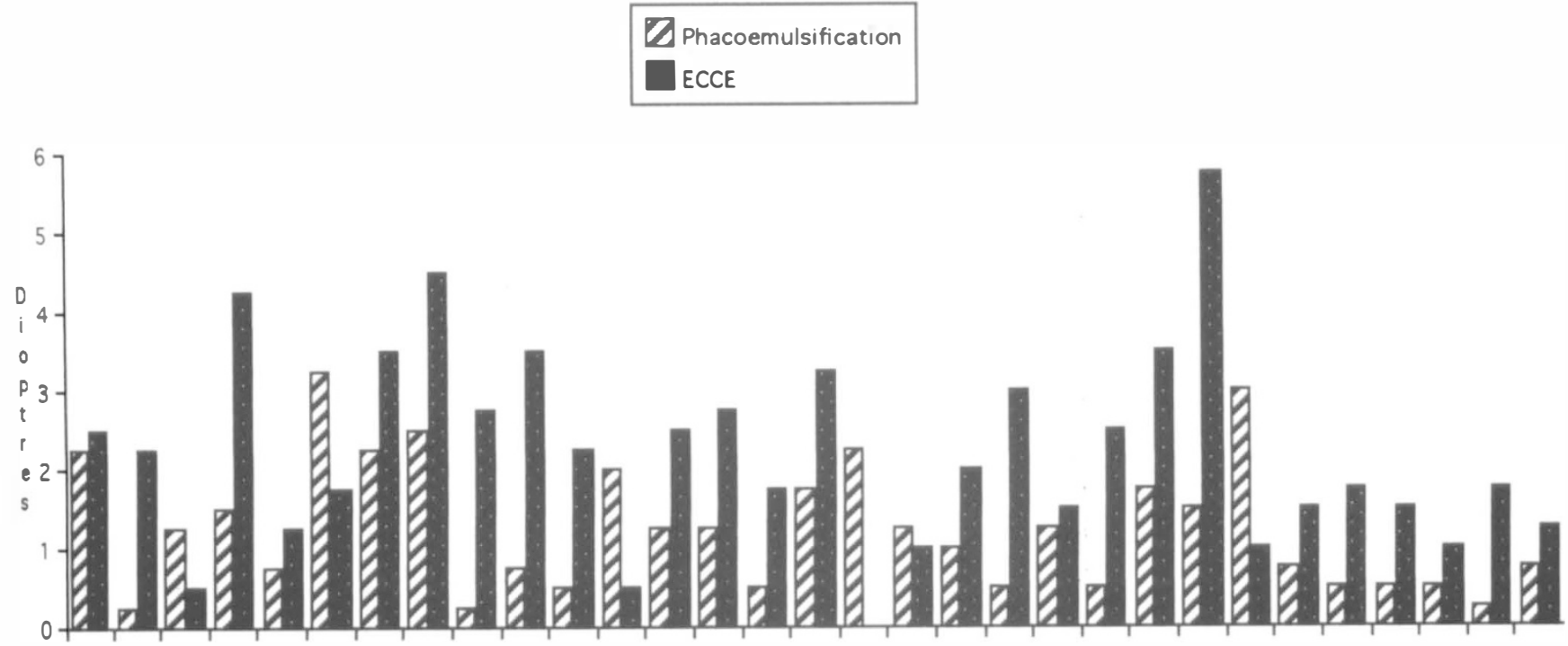

Fig. 4. Post-operative corneal cylindrical error without regard to axis for paired PE and ECCE eyes. 
undergoing PE (22 eyes; 70.9\%) (McNemar's test: $p<0.05$ ) (Fig. 4).

\section{DISCUSSION}

Several factors influence visual outcome and patient satisfaction following cataract surgery. These may be divided into independent predictors of visual outcome, disease-specific factors and generic healthstatus measures. Disease-specific factors are those directly related to the lens opacity such as Snellen visual acuity, functional impairment related to vision and cataract symptom score. Generic health-status measures comprise broad dimensions of healthrelated quality of life such as physical, emotional and social functioning. Finally, independent predictors of visual outcome include age and ocular comorbidity. ${ }^{7,8}$ In our study variability between subjects is eliminated and thus all the above factors are controlled. In addition, the viewing distance and level of illumination under which visual acuity is tested are identical for paired eyes.

It is not the aim of this paper to argue for or against SBCE but to provide a unique comparison of two different types of cataract surgery. Previous investigators have evaluated the visual and refractive results of PE and ECCE but not in the same patient over a single period of time. Consequently, matched pairs of eyes have not been studied and previous comparisons have been made only in terms of disease-specific factors.

In our study uncorrected visual recovery was similar for the two groups. This has not been the experience of other workers. ${ }^{1,2,4}$ A statistically similar proportion of PE and ECCE eyes achieved a satisfactory best corrected final visual acuity of $6 / 12$ or better, and our results are comparable to reports in the literature. ${ }^{9}$ However, an excellent final corrected visual acuity of $6 / 6$ or better was significantly more common among phacoemulsified eyes. This is consistent with the results of previous investigators, ${ }^{2}$ and is reflected in the greater patient satisfaction associated with this technique.

We recorded less astigmatism in PE eyes 24-53 weeks post-operatively than in their fellow eyes which underwent simultaneous conventional ECCE. The mean post-operative cylinder and the proportion of eyes with corneal astigmatism greater than $1.5 \mathrm{D}$ was significantly higher among eyes undergoing ECCE than among those operated on using the PE technique. The reduced astigmatism found among phacoemulsified eyes is in agreement with previous reports $^{2,3}$ and probably reflects the smaller size of the incision associated with this technique. . $^{1,10}$

In conclusion, the beneficial effects of phacoemulsification on astigmatism and visual outcome in the early post-operative period are confirmed in this unique cohort of patients.

Key words: Astigmatism, Cataract surgery, Endocapsular, Phacoemulsification.

\section{REFERENCES}

1. Neumann AC, McCarty GR, Sanders DR, Raanan MG. Small incisions to control astigmatism during cataract surgery. J Cataract Refract Surg 1989;15: 78-84.

2. Watson A, Sunderraj P. Comparison of small-incision phacoemulsification with standard extracapsular cataract surgery: post-operative astigmatism and visual recovery. Eye 1992;6:626-9.

3. Dam-Johansen M, Olsen T. Refractive results after phacoemulsification and ECCE: a comparative study. Acta Ophthalmol (Copenh) 1993;71:382-7.

4. Wishart PK, Austin MW. Combined cataract extraction and trabeculectomy: phacoemulsification compared with extracapsular technique. Ophthalmic Surg 1993;24:814-21.

5. Benezra D, Chirambo MC. Bilateral versus unilateral cataract extraction: advantages and complications. Br J Ophthalmol 1978;62:770-3.

6. Beatty S, Aggarwal RK, David DB, Guarro M, Jones H, Pearce JL. Simultaneous bilateral cataract extraction in the UK. Br J Ophthalmol 1995;79:1111-4.

7. Damiano AM, Steinberg EP, Cassard SD, Bass EB, Diener-West M, Legro MW, et al. Comparison of generic versus disease-specific measures of functional impairment in patients with cataract. Med Care 1995;33:120-30.

8. Schein OD, Steinberg EP, Cassard SD, Tielsch JM, Javitt JC, Sommer A. Predictors of outcome in patients who underwent cataract surgery. Ophthalmology 1995;102:817-23.

9. Powe NR, Schein OD, Gieser SC, Tielsch JM, Luthra R, Javitt J, Steinberg EP. Synthesis of the literature on visual acuity and complications following cataract extraction with intraocular lens implantation. Arch Ophthalmol 1994;112:239-52.

10. Levy JH, Pisacano AM, Chadwick K. Astigmatic changes after cataract surgery with a $5.1 \mathrm{~mm}$ and $3.5 \mathrm{~mm}$ sutureless incisions. J Cataract Refract Surg 1994;20:630-3. 